\title{
Aims and Scopes of the Special Issue: Foundations of Astrophysics and Cosmology
}

\author{
Salvatore Capozziello ${ }^{1}$. Tomislav Prokopec ${ }^{2}$. \\ Alessandro D. A. M. Spallicci ${ }^{3}$
}

Received: 23 February 2017 / Accepted: 1 March 2017 / Published online: 29 March 2017

(C) Springer Science+Business Media New York 2017

From the infinitesimal scale of particle physics to the cosmic scale of the universe, there have been spectacular advances in physics during the past century. The understanding of the universe is largely based on electromagnetic observations. As photons are the messengers, it is very important for fundamental physics to test the foundations of electromagnetism. The same stands for gravitation, the dominant interaction at large scale, for which we have just witnessed the opening of the high frequency gravitational wave window.

The basic properties of the Universe are often believed to be well understood, with only minor adjustments left in front of us. The observations which may represent a problem are interpreted to make them concordant or treated as peculiarities to be later dealt within the framework of an otherwise correct model. Further, assuming our current description of gravity and electromagnetism, we sometimes run into extremely complex and sophisticated cosmological models, partly based on purposely built "dark" or unknown ingredients. Ironically, we are awkwardly confronted with a largely unknown universe, and yet we adopt the term "precision cosmology".

Indeed, cosmology faces puzzling observations. First, galactic rotation curves, lensing and clustering do not appear to be generated by conventional baryonic matter.

Alessandro D. A. M. Spallicci

spallicci@cnrs-orleans.fr

Salvatore Capozziello

capozziello@unina.it

Tomislav Prokopec

t.prokopec@uu.nl

1 Università degli Studi di Napoli Federico II, Napoli, Italy

2 Universiteit Utrecht, Utrecht, The Netherlands

3 Université d'Orléans et Centre Nationale de la Recherche Scientifique, Orléans, France 
Second, it is commonly, although not universally, accepted that cosmic expansion is accelerating. We are clearly still facing aspects of the universe that we do not understand.

While the standard approach might lead to the ultimate answers as promised, concurrently the scientific community has to explore other avenues. Comparing alternative and standard views, it is the sole way that science can advance.

Thus, a debate on the foundations and on the theories of physics with a bearing on cosmology, or conversely a debate on cosmology (theoretical or observational) with a bearing on the foundations and theories of physics are the two tracks along which we collected these four contributions, while others may follow. In this issue, the papers deal with massive photons, the microwave background, the cosmological red shift and inflation. 